\title{
Evaluación de la eficiencia formativa de las universidades públicas colombianas 2003-2012 utilizando el análisis envolvente de datos [DEA]
}

\section{Training efficiency of Colombian public universities assessment 2003-2012 using the Data Envelopment Analysis [DEA]}

\author{
Gabriel Yáñez Canal \\ Doctor en ciencias, especialidad en matemática educativa \\ Universidad Industrial de Santander \\ gyanez@uis.edu.co \\ Ana Patricia García Amado \\ Licenciada en matemáticas \\ Universidad Industrial de Santander \\ anna.garciamado@gmail.com \\ Manuela González Hurtado \\ Licenciada en matemáticas \\ Universidad Industrial de Santander \\ manuelavatar@gmail.com
}

\section{Resumen}

Para cumplir con el requerimiento de inspección y vigilancia de las Instituciones de Educación Superior (IES), establecido en la Ley 30 de 1992 que regula la Educación Superior en Colombia, desde 2003 se inició el proceso de evaluación de las universidades públicas colombianas utilizando un conjunto de indicadores de gestión construidos por el Ministerio de Educación Nacional (MEN) en conjunto con el Sistema de Universidades Estatales (SUE). Con el ánimo de conocer el desarrollo que en los indicadores de formación han tenido las universidades en el periodo 2003-2012 se realizó un análisis de los resultados de los indicadores utilizando el Análisis Envolvente de Datos que mide en términos relativos el nivel de eficiencia de unidades organizacionales del mismo tipo. Se aplicó el modelo CCR orientado a los productos. Los resultados dan cuenta de un desarrollo sostenido de las universidades en general, así como también de los atrasos que tienen en algunos de los indicadores considerados.

Palabras clave: Indicadores de Gestión, Análisis Envolvente de Datos, Eficiencia, Universidades.

\begin{abstract}
In order to comply with the requirement of inspection and surveillance of Higher Education Institutions (IES acronym in Spanish), established in Act 30 of 1992 which governs Higher Education in Colombia, the assessment process of Colombian public universities began using a set of management indicators elaborated by the National Ministry of Education (MEN acronym in Spanish) together with the State University System (SUE acronym in Spanish). With the purpose of getting to know the development that the universities have had in the training indicators in the 2003-2012 period an analysis of the results of the indicators was done using the Data Envelopment Analysis which measures the level of efficiency of the organizational units of the same type in relative terms. The CCR model was applied oriented to the products. The results give evidence of an overall sustained development of the universities, as well as of the delays that they have in some of the indicators that were considered.
\end{abstract}

Key Words: Management indicators, Data Envelopment Analysis, Efficiency, Universities.

\section{Introducción}

La Educación Superior en Colombia está regulada, en términos generales, por las leyes 30 de 1992, 115 de 1994 , 715 de 2001, 749 de 2002, 1188 de 2008, 1324 de 2009 y 1740 de 2014. De estas, la ley 30 establece en los artículos 1 y 2 que "la Educación Superior es un servicio público cultural inherente a la finalidad social del Estado". Con fines de regulación, esta Ley, en los artículos 31 (adicionado por la L1740/2014, art. 1) y 32, fomenta la inspección y vigilancia de las Instituciones de Educación Superior (IES) a través de evaluaciones permanentes y 
bajo ciertos parámetros establecidos por el Ministerio de Educación Nacional (MEN) y el Sistema de Universidades Estatales (SUE). Para medir la eficiencia de las universidades públicas, SUE y MEN, desde 2003, se dieron a la tarea de construir un sistema de indicadores de gestión para evaluar el manejo de sus recursos y los logros obtenidos en sus misiones de formación, investigación y extensión. Desde ese momento hasta nuestros días la alianza SUE-MEN ha venido diseñando diferentes modelos para evaluar los resultados de los indicadores en las universidades públicas (Torres y Yáñez, 2012). Los modelos hasta ahora utilizados no permiten identificar las deficiencias de las universidades que no alcanzan los mayores niveles de eficiencia, en otras palabras, qué tanto, en términos relativos, deben mejorar los valores de sus indicadores para alcanzar niveles máximos de eficiencia.

Para suplir la carencia señalada, se realizó una investigación con una metodología que permitiera, además de analizar la eficiencia de las universidades a través del tiempo, identificar las deficiencias de las de menor rendimiento. El análisis dinámico de las eficiencias relativas de las universidades se efectuó en el período 2003-2012. La identificación de las deficiencias relativas en los aspectos analizados y el cálculo de los porcentajes de atrasos se realizaron con base en los datos de 2012, ya que en el momento de escribir este artículo no se conocían los resultados de los indicadores para el año 2013. Para lograr este objetivo se utilizó la técnica del Análisis Envolvente de Datos (AED), DEA en inglés: Data Envelopment Analysis. El análisis se realizó con base en modelos de eficiencia. Si bien los cálculos de las medidas de eficiencia se realizaron considerando el grupo de todas las universidades, para las comparaciones relativas entre ellas se consideró prudente formar grupos de universidades homogéneas de acuerdo a su nivel de capacidad disponible. En este documento se presentan los resultados y análisis relacionados exclusivamente con la misión de formación.

Este artículo tiene la siguiente estructura: En primera instancia se presentan los indicadores que miden la capacidad de las universidades y que se consideran como insumos en el modelo, y los indicadores que se refieren a la misión de formación, que son los productos del modelo. Acto seguido, y con el ánimo de comparar las universidades con sus pares semejantes, se conforman varios grupos de universidades de acuerdo a los indicadores de capacidad que han mostrado mayor consistencia a lo largo del tiempo. Posteriormente se realiza un breve resumen de la técnica AED, en el cual se exponen los conceptos y los modelos básicos necesarios que permitieron el análisis propuesto. Luego, se presenta un análisis de los resultados obtenidos para el período 2003-2012 y un análisis más detallado del año 2012. Finalmente, las conclusiones y la bibliografía referenciada.

\section{Indicadores}

El modelo subyacente a los indicadores de gestión creados por el SUE en asocio con el MEN, asume a las universidades como unidades de decisión que requieren de una cantidad de insumos para la producción de ciertos productos. El modelo contempla cinco grupos de indicadores relacionados con Capacidad, Formación, Investigación, Extensión y Bienestar Estudiantil. El grupo de capacidad corresponde a los insumos o entradas que las universidades requieren para su desempeño y los cuatro grupos restantes se asocian a indicadores de resultados o de producción. En este trabajo solo se analizaron los indicadores dirigidos a evaluar la gestión formativa de las universidades.

Los indicadores de capacidad, los insumos en el modelo AED, son los siguientes:

- Número de docentes de tiempo completo. Este indicador se calcula con base en el número de profesores de tiempo completo, de tiempo parcial, especiales o de cátedra. Para homologar los profesores de cátedra a tiempo completo se hace la equivalencia de un profesor de tiempo completo por cada 40 horas de cátedra.

- Recursos financieros. Este indicador comprende todos los recursos financieros obtenidos por la Universidad: los aportes del estado, los ingresos por matrícula y por actividades de extensión.

- Gastos administrativos. Este indicador da cuenta de todos los gastos de personal no docente en los que incurren las universidades.

- Metros cuadrados construidos. Este indicador se calcula con base en la cantidad de metros cuadrados dedicados por las universidades a sus actividades docentes, investigativas $y$ administrativas.

En este trabajo utilizamos como insumos los indicadores de docentes, gastos administrativos y los metros cuadrados construidos. A pesar de toda su importancia, se tomó la decisión de excluir el indicador de recursos financieros, ya que un análisis de los datos reportados por el MEN para el periodo 2003-2012 y que aparecen en el SNIES ${ }^{1}$, mostró demasiados valores que no se corresponden con el tamaño de las universidades y que no se compadecen para nada con los de los otros indicadores de capacidad considerados.

Los indicadores de formación utilizados, y que son los productos en el AED, son los siguientes:

- Programas de Pregrado (PPRE). Mide la oferta de programas académicos de formación de pregrado.

- Programas de Posgrado (PPOS). Hace referencia al número de programas académicos en el nivel de formación de posgrado. La información se desagrega por nivel de formación: especialización, especialidad médica, maestría y doctorado, por metodología (presencial o distancia) con 
ponderaciones diferentes acorde a sus niveles de complejidad.

- Matrículas en pregrado (MPRE). Da cuenta del número de estudiantes matriculados en programas de pregrado. Dado que la población estudiantil puede variar de semestre a semestre y que las ceremonias de graduación se llevan a cabo en fechas diferentes a lo largo del año, este indicador reporta el máximo número de estudiantes que está matriculado en el año, el que registra la mayor cobertura.

- Matriculados en programas de posgrado (MPOS). Se refiere al número de estudiantes matriculados en programas de maestría y doctorado. Se reporta el máximo número de estudiantes que están matriculados en el año.

- Graduados en pregrado (GPR). Es el número de graduados en programas de pregrado. La información se pondera dependiendo de la modalidad de enseñanza.

- Graduados en posgrado (GPOS). Es el número de graduados en programas de posgrado. En los programas curriculares de maestría y doctorado ofrecidos mediante convenios entre varias universidades, la institución dueña del registro debe reportar los graduados. En los programas ofrecidos en red por instituciones que tienen registro SNIES, estas deben reportar sus graduados respectivos.

- Estudiantes de primer nivel (PRIM). semestre. Este indicador da cuenta del número de estudiantes nuevos matriculados en primer curso en el período de referencia en programas curriculares conducentes a título. Se toma el total del año y se ponderan de acuerdo a la metodología de enseñanza (presencial o distancia) y al tipo de programa.

- ECAES o Pruebas Saber-PRO. Da cuenta del número de estudiantes con resultados en la prueba ECAES por encima del percentil 75 . El percentil 75 se calcula con base en toda la población que presenta el examen del área respectiva, incluye universidades públicas y privadas.

Dadas las diferencias que en costo existen entre los diversos programas que ofrecen las universidades públicas, bien por las especificidades propias de cada programa como por el tipo de metodología (presencial, semi presencial o distancia), y dada la estrecha relación que existe entre los recursos y los productos en el modelo AED, se hace necesario ponderar los diferentes indicadores teniendo en cuenta estas diferencias. Dada la inexistencia en el país de un estudio claro de costos universitarios, el MEN-SUE optó por utilizar la ponderación que utilizan los australianos. Estas ponderaciones se utilizaron para calcular todos los indicadores de formación, excepto el relacionado con las Pruebas Saber-PRO.

\section{Grupos de Universidades}

Para realizar el análisis de la eficiencia formativa, se consideró conveniente clasificar a las universidades en seis grupos de acuerdo a los indicadores de capacidad descritos previamente. Se utilizó el análisis de conglomerados, específicamente el método Ward con distancia euclidiana al cuadrado, utilizando los valores de los tres indicadores de capacidad, descritos previamente, en el año 2012. La clasificación anterior se modificó con base en la variable profesores para evitar grupos demasiado grandes o demasiados grupos unitarios.

De esta forma se conformaron seis grupos de universidades tal como se muestran en la Tabla 1.

Tabla 1. Clasificación de las universidades de acuerdo a su capacidad disponible.

\begin{tabular}{|c|c|c|c|c|}
\hline \multicolumn{5}{|c|}{ UNIVERSIDADES PÚBLICAS COLOMBIANAS } \\
\hline \multicolumn{2}{|c|}{ MUY PEQUEÑAS } & \multicolumn{2}{|c|}{ MEDIANAS BAJAS } & MUY GRANDES \\
\hline \multicolumn{2}{|c|}{$\begin{array}{c}\text { Pacifico } \\
\text { Ocaña }\end{array}$} & $\begin{array}{c}\text { Surcolombiana } \\
\text { Córdoba } \\
\text { Magdalena }\end{array}$ & $\begin{array}{c}\text { Militar } \\
\text { Cundinamarca } \\
\text { Quindio }\end{array}$ & $\begin{array}{l}\text { Antioquia } \\
\text { Nacional }\end{array}$ \\
\hline \multicolumn{2}{|c|}{ PEQUEÑAS } & \multicolumn{2}{|c|}{ MEDIANAS } & GRANDES \\
\hline $\begin{array}{c}\text { Sucre } \\
\text { Llanos } \\
\text { Guajra } \\
\text { Popular } \\
\text { Nariño }\end{array}$ & $\begin{array}{l}\text { Cucuta } \\
\text { Colegio } \\
\text { Chocó } \\
\text { Amazonia }\end{array}$ & $\begin{array}{l}\text { Cartagena } \\
\text { Caldas } \\
\text { Pedagogica } \\
\text { Tolima }\end{array}$ & $\begin{array}{c}\text { Pereira } \\
\text { Pamplona } \\
\text { Allántico } \\
\text { UNAD }\end{array}$ & $\begin{array}{c}\text { Cauca } \\
\text { UPTC } \\
\text { UIS } \\
\text { Valle } \\
\text { Distrital }\end{array}$ \\
\hline
\end{tabular}

Un primer grupo de universidades Muy Pequeñas (MP) conformado por Pacífico y UFPS-seccional Ocaña ${ }^{2}$. Un segundo grupo, que se denomina Pequeñas $(P)$, conformado por las universidades Sucre, Guajira, Nariño, Colegio Mayor, Amazonia, Llanos, Popular, Cúcuta y Chocó. El tercer grupo conformado por las universidades de tamaño medio-bajo, nombradas Medianas Bajas (MB) formado por las universidades Surcolombiana, Magdalena, Cundinamarca, Córdoba, Militar y Quindío. El cuarto grupo de universidades de tamaño medio denominado Medianas (M) constituido por las universidades Pedagógica, Pereira, Caldas, Atlántico, Cartagena, Pamplona, Tolima y UNAD. El quinto grupo constituido por las universidades grandes, que se denomina Grandes $(G)$, conformado por las universidades del Valle, UIS, UPTC, Distrital y Cauca. Finalmente el sexto grupo de las Muy Grandes (MG) conformado por la Nacional y Antioquia, con la salvedad de que la Nacional es mucho mayor que la de Antioquia.

\section{Análisis Envolvente de Datos (AED)}

El AED ha venido siendo usado para evaluar la eficiencia tanto de instituciones de educación básica (Fuentes Pascual, 2000; Liu et al. 2011) como de educación superior (Bougnol y Dulá, 2006; Abbott y

1 - SNIES - Sistema Nacional de Información de la Educación Superior

http://www.mineducacion.gov.co/sistemasdeinformacion/1735/w3-propertyname-2672.htm

2 - La seccional de Ocaña de la Universidad Francisco de Paula Santander, con sede principal en Cúcuta, es la única seccional que tiene presupuesto propio asignado directamente por el gobierno nacional. Por esta razón se considera como una universidad pública más. 
Doucouliagos 2003; Johnes, 2006; Kuah y Wong, 2011). Se ha utilizado para evaluar aspectos como la eficiencia investigativa de instituciones de educación superior (Jones y Yu 2008), la eficiencia de la enseñanza en educación superior (Johnes, 2006; Beasley, 1995), la producción académica (Soto et al. 2005) y la eficiencia de grupos de investigación (Pino et al. 2010), entre otros.

El Análisis Envolvente de Datos (AED), desarrollado por Charnes, Cooper y Rhodes (1978, 1981), es un método no paramétrico y determinístico de evaluación de la eficiencia relativa de un grupo de unidades productivas homogéneas, que en este trabajo identificamos como unidades de decisión: UD. Mediante técnicas de programación lineal, y con base en valores observados de insumos y productos, construye una frontera eficiente que permite evaluar la eficiencia de las unidades consideradas. Tal frontera está conformada por aquellas unidades que son eficientes $y$, como el nombre envolvente lo indica, "envuelve" o limita a aquellas que no lo son. De esta forma, el AED es una herramienta que da respuesta a la pregunta de cómo medir la eficiencia relativa identificando las unidades más eficientes y proponiendo mejoras, tanto en los insumos como en los productos, a las unidades que no se encuentran en la frontera eficiente.

En el caso en que se tengan en cuenta $\boldsymbol{m}$ insumos y $\boldsymbol{r}$ productos la medida de eficiencia estará dada por una razón entre los productos producidos y los insumos requeridos expresados como combinación lineal, es decir, son sumas de productos e insumos ponderados adecuadamente. Esta razón se interpreta como una medida de la cantidad de productos producidos por unidad de insumos, tal como lo muestra la siguiente expresión:

$$
\operatorname{eficiencia}(\theta)=\frac{\sum_{r=1}^{s} u_{r} y_{r}}{\sum_{i=1}^{m} v_{i} x_{i}}
$$

Donde

$$
\begin{aligned}
& y_{r}=\text { cantidad del producto } \boldsymbol{r} \\
& \boldsymbol{u}_{r}=\text { peso o ponderación asignada al producto } \boldsymbol{r}, \\
& \boldsymbol{r}=1,2, \ldots, s \\
& \boldsymbol{x}_{\boldsymbol{i}}=\text { cantidad del insumo } \boldsymbol{i}, \boldsymbol{i}=1,2, \ldots, m \\
& \boldsymbol{v}_{\boldsymbol{i}}=\text { peso o ponderación asignada al insumo } \boldsymbol{i}
\end{aligned}
$$

El asunto es determinar los pesos o ponderaciones $\boldsymbol{u}_{\boldsymbol{r}}$ de los productos y las ponderaciones $v_{i}$ de los insumos para poder calcular la eficiencia. Estas ponderaciones serán las que produzcan la mayor eficiencia de la UD que se esté considerando, es decir, se trata de resolver el siguiente problema fraccional:

$$
\left(P F_{o}\right){ }_{v, u}^{\max } \theta=\frac{u_{1} y_{1 o}+u_{2} y_{2 o}+\cdots+u_{s} y_{s o}}{v_{1} x_{1 o}+v_{2} x_{2 o}+\cdots+v_{m} x_{m o}}
$$

Sujeto a,

$$
\begin{array}{r}
\frac{u_{1} y_{1 j}+u_{2} y_{2 j}+\cdots+u_{s} y_{s j}}{v_{1} x_{1 j}+v_{2} x_{2 j}+\cdots+v_{m} x_{m j}} \leq 1 \\
(j=1, \ldots, n) \\
v_{1}, v_{2}, \ldots, v_{m} \geq 0 \\
u_{1}, u_{2}, \ldots, u_{s} \geq 0
\end{array}
$$

Las expresiones (2)-(5) se conocen como modelo AEDCCR, (CCR hace referencia a los autores: Charnes, Cooper y Rhodes) para la unidad de decisión $U D_{o}, o=1, \ldots, n$, cuando se tienen $\boldsymbol{n}$ Unidades de Decisión: $\mathrm{UD}_{1}, \mathrm{UD} 2, \ldots$, UDn y varios tipos de insumos $\mathrm{x} y$ productos y. El objetivo es maximizar la medida de eficiencia encontrando el mejor conjunto de pesos para las entradas $\left(v_{-} i, i=1, \ldots, m\right)$ y para las salidas $\left(\mathrm{u}_{-} \mathrm{r}, \mathrm{r}=1, \ldots, \mathrm{s}\right)$ para cada unidad.

El modelo anterior da cuenta de las características básicas de la medida de eficiencia a que da lugar la solución de este modelo:

- Para cada UD, AED - CCR forma insumos y productos virtuales, en el sentido de que a partir de los datos (insumos y productos) proporcionados por las unidades, el modelo selecciona el mejor conjunto de pesos $\left(v_{-} i^{\wedge^{*}}\right), \mathbf{i}=1,2, \ldots \mathrm{m}$ y $\left.\left(\mathbf{u}_{-} \mathbf{r}^{\wedge^{\star}}\right)^{\star}\right)$ $r=1,2, \ldots s$ que se le deben asignar a los insumos y productos, respectivamente, para mejorar su rendimiento. El conjunto de pesos difiere en cada una de las unidades, por lo que se tienen n modelos diferentes, uno para cada unidad de decisión. AED zanja la discusión alrededor de la importancia de los insumos o de los productos seleccionados, asignándolos de tal forma que se obtenga la mayor cantidad de productos como respuesta de la mejor combinación de los insumos, así para cada una de las unidades de decisión.

- Las expresiones (2) y (3) indican que las ponderaciones no se definen a priori, al contrario, dependen de los valores observados de todas las UD consideradas y se calculan para obtener el máximo valor de eficiencia.

- La restricción (3) significa que la proporción salidas virtuales/entradas virtuales no debe exceder de 1 para cada una de las UDs, incluyendo la que se está evaluando, por lo que el valor máximo factible para la eficiencia de cualquier unidad de decisión es $?^{\wedge^{*}=1}=1$. Es decir, esta restricción fija en 1 la relación máxima entre los productos generados respecto a los insumos utilizados en todas las unidades de decisión. Los pesos se calculan para cada UD de tal forma que no exista otra UD que con las mismas ponderaciones sobrepase el valor 1. En otras palabras, se asume como la máxima producción una unidad de productos (combinación lineal de 
todos los productos considerados) para cualquier asignación de los insumos utilizados para producirlas.

- Una UD debe obtener un valor de eficiencia 1 para ser considerada como eficiente.

- El subíndice o indica que las ponderaciones para los insumos y los productos son propios de cada UD, razón por la cual existen tantos modelos CCR como UD en consideración.

- El AED es una técnica no paramétrica ya que no especifica a priori ninguna forma funcional para la frontera de eficiencia; tampoco es estadística porque no presupone una distribución probabilística para la eficiencia no explicada.

La flexibilidad de los ponderadores evita la realización de juicios de valor por parte del investigador referentes a la importancia relativa de cada variable en la determinación de la eficiencia de una DMU, lo que en el caso de las universidades implicaría un trabajo desgastante y bien difícil de consensuar. Como suele suceder en la realidad, las ventajas, también, son muchas veces desventajas: el programa puede asignar una ponderación nula o muy escasa a un determinado factor que, desde el punto de vista teórico, tenga una gran importancia en la eficiencia relativa de las UD, como lo destaca Fuentes (2000). Para evitar esta situación se corrige el modelo exigiendo que los coeficientes a calcular sean estrictamente positivos (restricciones (4) y (5)).

El problema fraccional definido por las expresiones (2)-(5) tiene infinitas soluciones y no es un modelo lineal. Para eliminar estos problemas se propone un problema de programación lineal equivalente, con solución única, que toma la forma:

$$
\max _{u, v} \theta=\mu_{l} y_{1 o}+\cdots+\mu_{s} y_{s o}
$$

Sujeto a,

$$
\begin{gathered}
v_{1} x_{1 o}+\cdots+v_{m} x_{m o}=1 \\
\mu_{1} y_{1 j}+\cdots+\mu_{l} y_{l j} \leq v_{1} x_{1 j}+\cdots+v_{m} x_{m j} \\
(j=1, \ldots, n) \\
v_{1}, v_{2}, \ldots, v_{m} \geq 0 \\
u_{1}, u_{2}, \ldots, u_{s} \geq 0
\end{gathered}
$$

En este modelo, una UD se considera CCR - eficiente si ?_o $0^{\wedge^{*}}=1$ y existe al menos un óptimo con $?^{\wedge \star}>0, y$ donde son los vectores conformados por los pesos de los productos y de los insumos respectivamente; de lo contrario, es ineficiente. Si una UD es CCR - ineficiente, ?_ $\mathrm{O}^{\wedge^{*}<1}$ para un óptimo de lo que se sigue que se debe tener al menos una UD que satisfaga la igualdad:

$$
\sum_{r=1}^{s} \mu_{r}^{*} y_{r j}=\sum_{i=1}^{m} v_{i}^{*} x_{i j}
$$

Es decir, existe otra UD que alcanza eficiencia 1 con los mismos ponderadores que le permiten a la unidad analizada alcanzar su máximo, esto es, existe otra unidad con mayor eficiencia que la analizada ponderando de igual forma los insumos y los productos. Esta unidad forma parte de la frontera envolvente y se constituye en referente de la unidad en análisis.

Asociado al modelo de programación lineal definido en las ecuaciones (6) - (10) existe un modelo dual dirigido a los insumos y otro dirigido a los productos. Como en las universidades, salvo el gasto administrativo, los demás insumos considerados en este trabajo (número de docentes y metros cuadrados construidos) no tienen sentido reducirlos, se utiliza el modelo dual dirigido a los productos. El modelo dual orientado a los productos para el problema de programación lineal dado previamente tiene la forma siguiente:

$$
\left(P D_{o}\right) \quad \max _{\phi, \lambda} \phi
$$

Sujeto a,

$$
\begin{array}{r}
\phi y_{r o} \leq \sum_{j=1}^{n} \lambda_{j} y_{r j} \quad(r=1, \ldots, s) \\
\sum_{j=1}^{n} \lambda_{j} x_{i j} \leq x_{i o} \quad(i=1, \ldots, m) \\
\lambda_{j} \geq 0
\end{array}
$$

$\phi_{0}$ : Parámetro que mide la eficiencia de la unidad evaluada.

$\lambda_{j}$ : Ponderaciones obtenidas como solución del programa. Expresan el peso que posee cada UD dentro del grupo de comparación de la ?UD?_o.

En este modelo se compara la ?UD?_o con todas las UD reales o ficticias que produzcan lo mismo o más que ella y consuman menos o lo mismo que la ?UD?_o.

Así que si es igual a uno entonces la ?UD?_o será eficiente ya que el programa habrá buscado entre las UD reales y ficticias que produzcan lo mismo o más y usen la misma cantidad o menos insumos.

Este modelo pretende la maximización de la producción sin modificar el número de insumos que se utiliza actualmente. Se definen las holguras $? \wedge\left(-\wedge^{*}\right), ? \wedge\left(+\wedge^{*}\right)$ asociadas al valor óptimo como las diferencias no negativas de las expresiones (13) y (14):

$$
\begin{gathered}
\delta^{-^{*}}=Y \lambda-\phi y_{r o} \\
\delta^{+^{*}}=x-\lambda X
\end{gathered}
$$


Las holguras $? \wedge\left(-\wedge^{*}\right), ? \wedge\left(+\wedge^{*}\right)$ se refieren al exceso en los productos y los déficits en los insumos, respectivamente, respecto a la frontera constituida por algunas de las restantes unidades de decisión.

La solución óptima al modelo dual orientado a los productos, expresiones $12-15$, será ?(?? $\wedge^{\star}, ? \wedge^{\star \star}, ?{ }^{\wedge}\left(-\wedge^{\star}\right.$

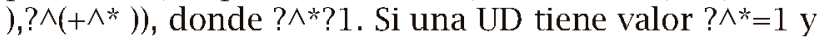

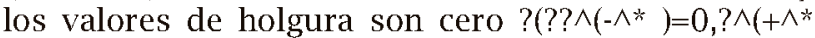
) $=0$ ), se dirá que dicha UD es técnicamente eficiente, de lo contrario será ineficiente.

Cuando se tienen unidades eficientes no se logra identificar cuál de ellas es de mayor eficiencia, siendo su valor de eficiencia uno, contrario a las unidades ineficientes que pueden ser ordenadas de acuerdo al valor que obtengan.

En este trabajo la intención no es dirigir la atención solo a las unidades ineficientes sino que también a las eficientes, por lo que los resultados obtenidos estarán bajo los modelos CCR, orientado a los productos y con el modelo de eficiencia.

\section{Análisis de los resultados}

La información de cada uno de los indicadores de gestión utilizados en esta investigación en el periodo de tiempo analizado fue tomada del SNIES

\section{Supereficiencia formación 2003-2012}

Las universidades cuyos valores de supereficiencia están por debajo de 1 se consideran ineficientes, y por encima de uno, técnicamente eficientes. Cabe destacar a las universidades Pacífico, UFPS-Cúcuta, Militar, Tolima y Distrital como las únicas que han logrado ser eficientes en todo el período evaluado. Dentro de estas universidades, sobresale significativamente Militar ya que en este período su valor de supereficiencia osciló entre 2,93 y 5,42 .

A continuación se presenta un breve análisis de la dinámica que han presentado las universidades respecto a su eficiencia en el período considerado. El análisis se realiza por grupos de universidades.

\section{Universidades Muy Pequeñas (MP)}

La Universidad del Pacífico se muestra eficiente en todo el período evaluado, sus mayores valores los alcanzó en los años 2007 y 2008 para luego descender hasta alcanzar en el año 2012 una eficiencia de 1,21, semejante a la obtenida en 2003. UFPS - Ocaña fue eficiente en los años 2005-2010 y posteriormente en el año 2012. (Ver Figura 1).

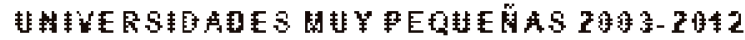

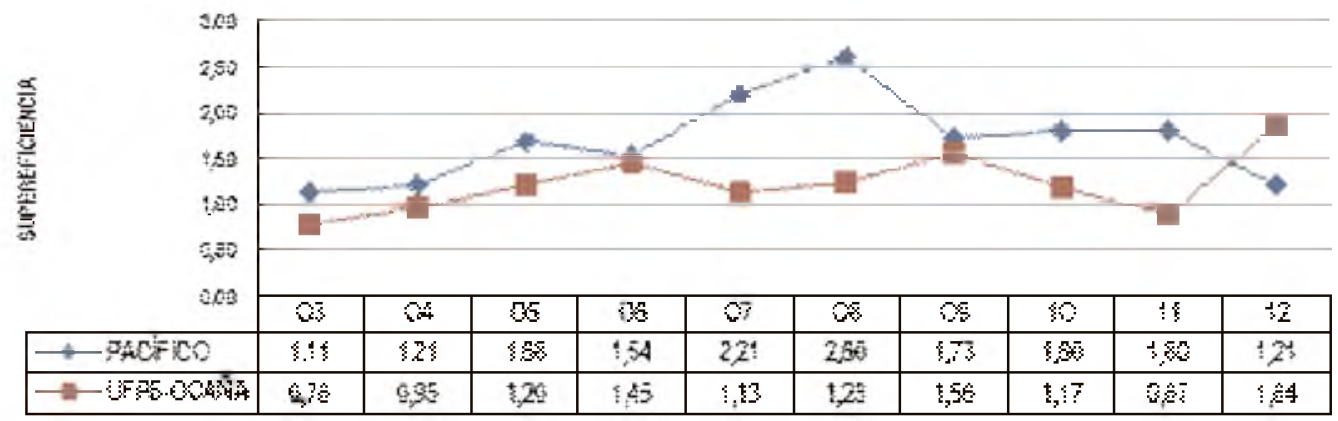

Figura 1. Supereficiencia periodo 2003-2012 Universidades Muy Pequeñas.

\section{Universidades Pequeñas $(P)$}

En la Figura 2 se observa que solo UFPS - Cúcuta es eficiente en todo el período evaluado, aunque otras universidades se han mostrado eficientes de manera muy significativa en algún año: Sucre (2004) 3,79; Popular (2007) 2,33, Nariño (2011) 1,76 y Guajira (2003) 1,72.

En 2012 solo Guajira y la UFPS_Cúcuta alcanzaron el nivel de eficiencia; Popular y Nariño, sin alcanzar la eficiencia, presentaron valores razonablemente altos; Chocó, Sucre y el Colmayor obtuvieron rendimientos medios en tanto que Llanos y Amazonas presentaron valores deficientes. 


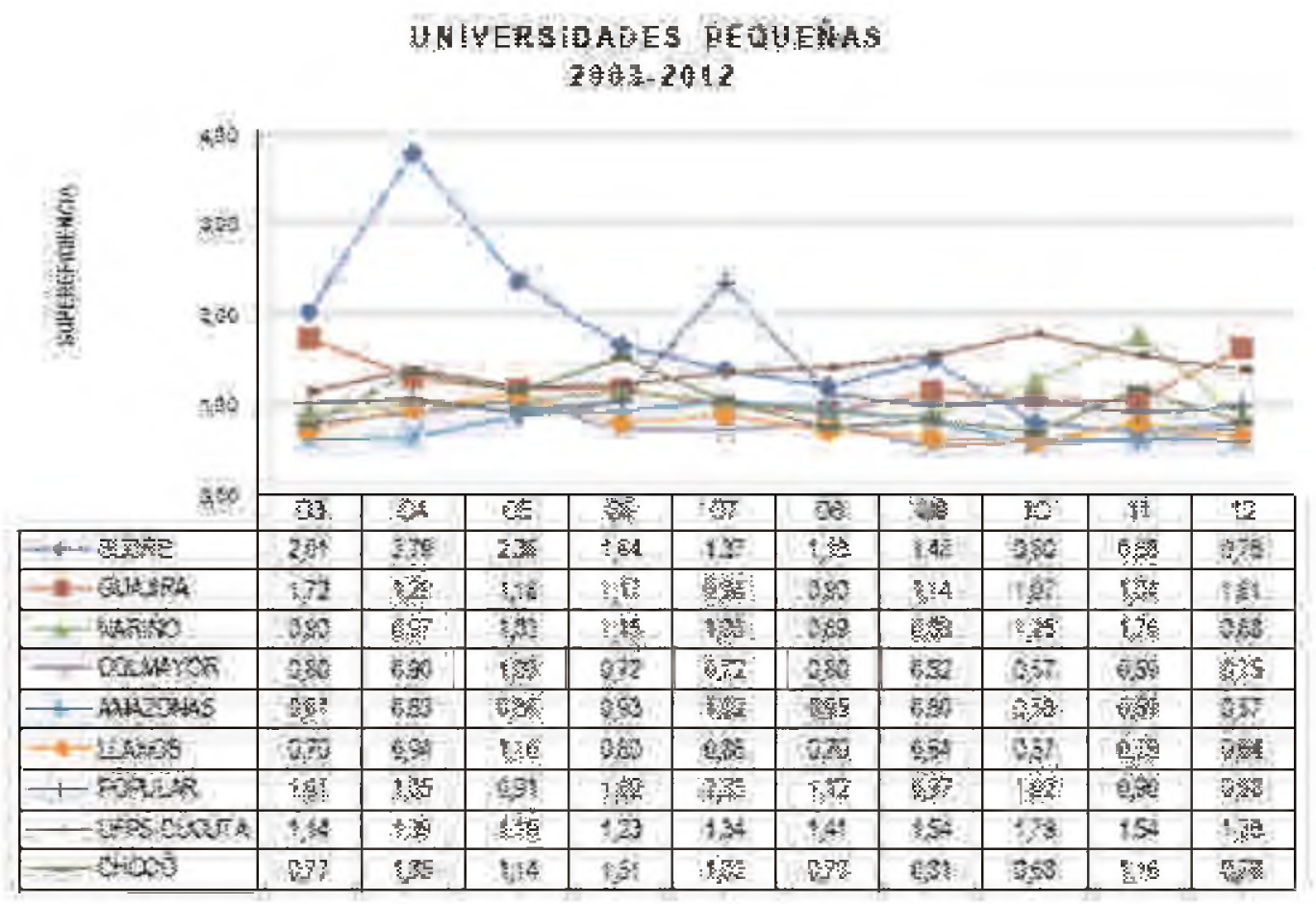

Figura 2. Supereficiencia periodo 2003-2012 Universidades Pequeñas.

\section{Universidades Medianas Bajas (MB)}

En la Figura 3 se observa que la Universidad Militar sobresale en este grupo, no solo ha sido eficiente todos los años sino que también ha sido la más eficiente de todas. En 2008 alcanzó un valor de 5,42 el más alto de todo el sistema en el periodo de tiempo considerado. Magdalena es otra que se destaca, ya que solo en 2010 no se muestra eficiente $(0,88)$. Cundinamarca presentó niveles de eficiencia relativamente estables alrededor de 0.95 en los primeros 6 años para luego estar alrededor de 0,62 en los últimos cuatro años.

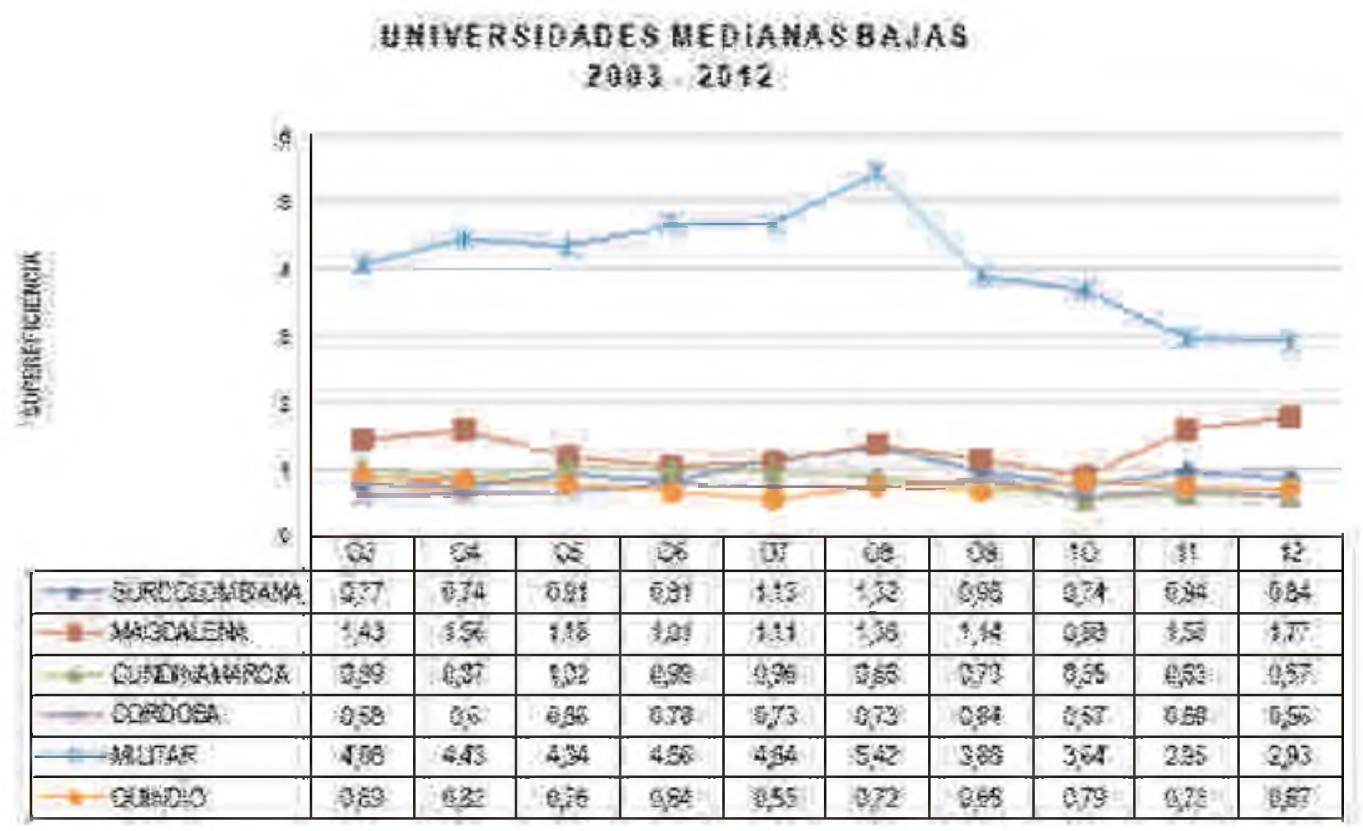

Figura 3. Supereficiencia periodo 2003-2012 Universidades Medianas Bajas. 
La Surcolombiana presentó niveles de eficiencia en 2007 y 2008 como resultado de un proceso de crecimiento en los años anteriores para luego iniciar otro de decrecimiento en los últimos cuatro años con un valor medio de 0.87. Córdoba y Quindío no han logrado ser eficientes en ningún año del período evaluado, con valores medios de eficiencia de 0,67 y 0,72 respectivamente.

\section{Universidades Medianas (M)}

Como se puede ver en la Figura 4 en este grupo se destaca Tolima, evaluada como eficiente en todos los años 2003-2012, con valores entre 1.66 y 2.51. Pamplona salvo en $2011(0,97)$, en los demás años alcanzó la eficiencia, valores entre 1.03 y 1.68. La UNAD alcanzó la eficiencia en 8 de los 10 años considerados, el promedio de sus valores es 1,43 .

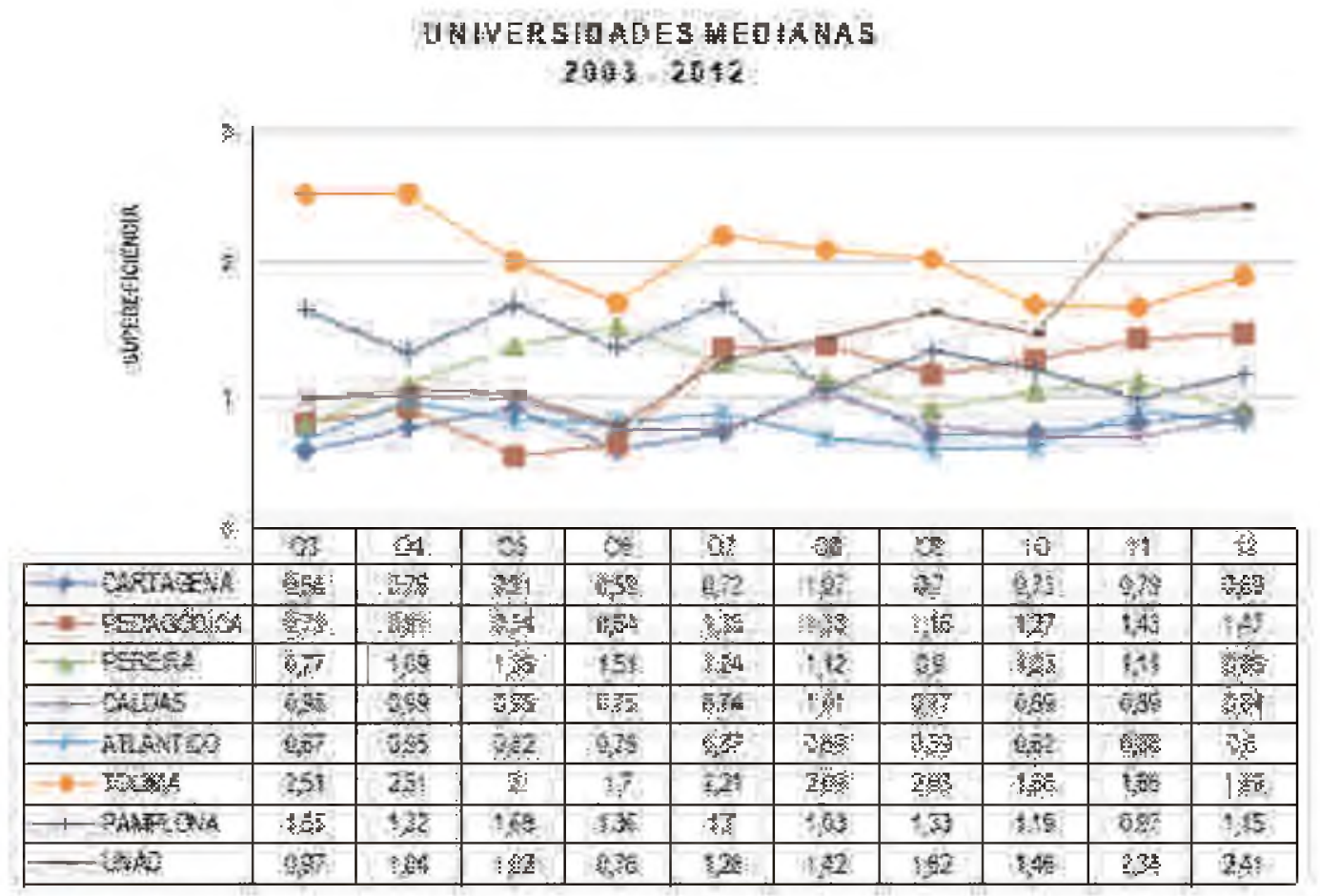

Figura 4. Supereficiencia periodo 2003-2012 Universidades Medianas.

Como se puede ver en la Figura 4 en este grupo se destaca Tolima que ha sido eficiente en todos los años 2003-2012. Pamplona alcanzó niveles de eficiencia en todos los años salvo en $2011(0,97)$.

La Pedagógica, si bien los primeros años mostró niveles de no eficiencia, a partir de 2007 ha logrado ser eficiente con valores entre 1,16 y 1,47. La Tecnológica de Pereira ha logrado ser eficiente en 7 de los 10 años en estudio, sus valores oscilan entre 0,77 en 2003 y 1,51 en 2006. Caldas solo logró la eficiencia en 2008, los valores de eficiencia en los demás años se mueven entre 0.69 y 0.99 . Atlántico no logró ser eficiente en ninguno de los años considerados, sus valores han estado entre 0.59 y 0.95 , en 2012 alcanzó una eficiencia de 0,80 .

\section{Universidades Grandes (G)}

En la Figura 5 se muestra que Distrital es la universidad de mejor desempeño en este grupo a lo largo del tiempo, ha sido la única eficiente en todo el periodo. No muy lejos se encuentra la Universidad del Valle que solo ha sido ligeramente ineficiente al inicio y al final del periodo, en 2012 alcanza un nivel de eficiencia de 0.97 . UIS y Cauca muestran un comportamiento decreciente en el todo el periodo, más llamativo el de la UIS que fue eficiente en los años 2003-2006 pero que con excepción de 2010 no volvió a serlo, en 2012 alcanzó una eficiencia medianamente alta de 0,88 . La UPTC presentó valores muy bajos en todo el periodo con excepción de 2011 que casi logra ser eficiente $(0,96)$; excluido este año sus valores oscilaron entre 0.56 y 0.74 . 


\section{GHIVEASIDAEES GAA DAES}

$204-2012$

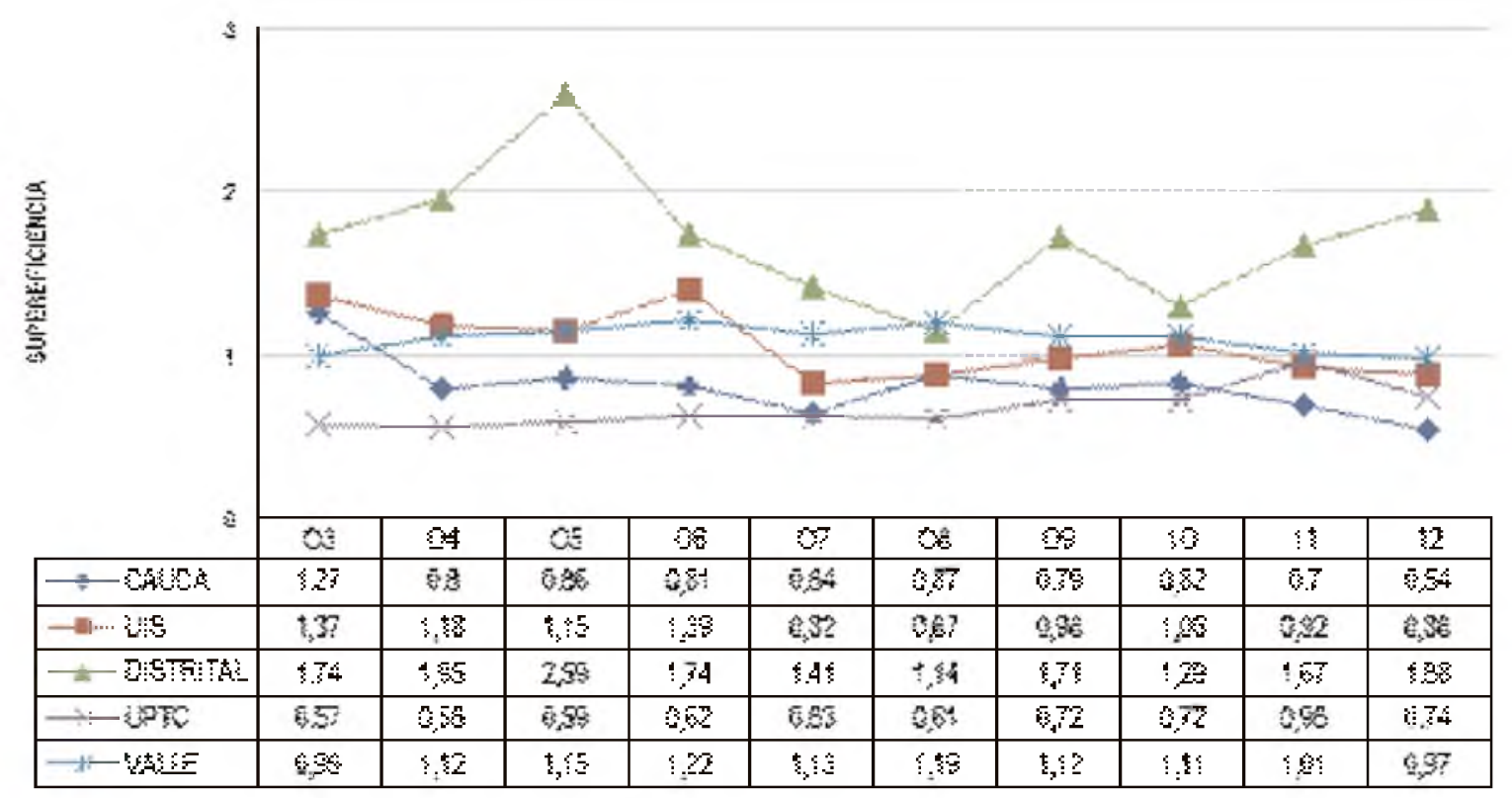

Figura 5. Supereficiencia periodo 2003-2012 Universidades Grandes.

\section{Universidades muy Grandes (MG)}

La Figura 6 da cuenta de cómo las dos integrantes de este grupo (Antioquia y Nacional) muestran un comportamiento oscilatorio en sus niveles de eficiencia, ninguna de las dos logró ser eficiente en todo el período: Antioquia lo logró en 5 ocasiones y la Nacional en tres.

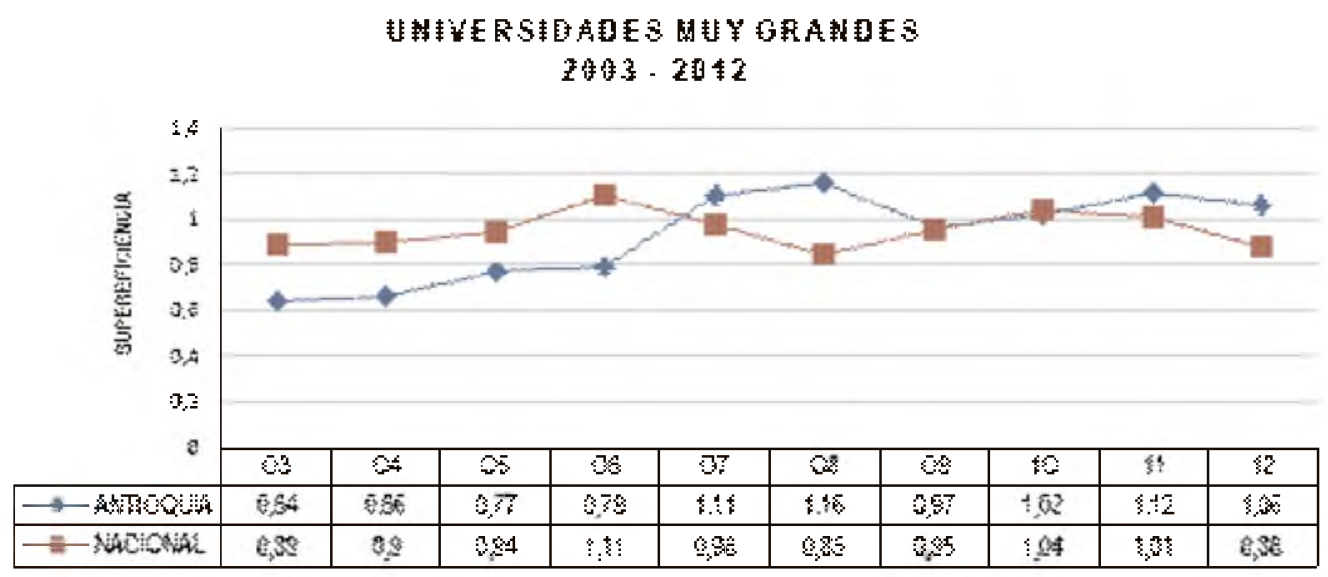

Figura 6. Supereficiencia periodo 2003-2012 Universidades Muy Grandes.

El rendimiento de la Universidad de Antioquia está claramente diferenciado: en los primeros cuatro años mostró niveles bajos de eficiencia (media de 0.72) en tanto que en los últimos 6 años su nivel fue alto (media de 1.07). La Nacional en sus resultados es mucho más homogénea oscilando entre 0,85 y 1,11 con media de 0,96.

\section{Análisis año 2012}

A continuación se presenta el análisis de la supereficiencia alcanzada por cada una de las universidades en el índice de formación para el año 2012. El estudio corresponde al año mencionado ya que hasta la fecha es la información más actualizada suministrada por el SUE y el MEN y, por ende, la más próxima al estado actual de las 
universidades, lo que permite dar cuenta con mejor acierto de cómo están hoy las universidades en términos de eficiencia en el índice de formación y cuáles son los aspectos específicos que deben mejorar para ser eficientes.

En la Tabla 2 se presentan, en orden descendente, los resultados de supereficiencia para el año 2012 en el índice de formación. Se identifican las universidades de acuerdo a su capacidad de la siguiente forma: MP: Muy Pequeñas; P: Pequeñas; MB: Medianas-Pequeñas; M: Medianas; G: Grandes y MG: Muy Grandes.

Tabla 2. Clasificación de las IES por supereficiencia en el índice de formación 2012.

\begin{tabular}{c|l|c|c|l|c}
\hline Posición & \multicolumn{1}{|c|}{ IES } & \multicolumn{1}{|c|}{ SE* $^{*}$} & Posición & & IES \\
\hline 1 & MILITAR & 2,93 & 17 & NARIÑO & 0,88 \\
\hline 2 & UNAD & 2,41 & 18 & NACIONAL & 0,88 \\
\hline 3 & TOLIMA & 1,89 & 19 & UIS & 0,88 \\
\hline 4 & DISTRITAL & 1,88 & 20 & SURCOLOMBIANA & 0,84 \\
\hline 5 & UFPS-OCAÑA & 1,84 & 21 & CALDAS & 0,84 \\
\hline 6 & MAGDALENA & 1,77 & 22 & ATLÁNTICO & 0,80 \\
\hline 7 & GUAJIRA & 1,61 & 23 & CHOCÓ & 0,79 \\
\hline 8 & PEDAGÓGICA & 1,47 & 24 & SUCRE & 0,78 \\
\hline 9 & UFPS-CÚCUTA & 1,38 & 25 & COLMAYOR & 0,75 \\
\hline 10 & PACIFICO & 1,21 & 26 & UPTC & 0,74 \\
\hline 11 & PAMPLONA & 1,15 & 27 & QUINDIO & 0,67 \\
\hline 12 & ANTIOQUIA & 1,06 & 28 & LLANOS & 0,64 \\
\hline 13 & POPULAR & 0,98 & 29 & AMAZONAS & 0,57 \\
\hline 14 & VALLE & 0,97 & 30 & CUNDINAMARCA & 0,57 \\
\hline 15 & CARTAGENA & 0,89 & 31 & CORDOBA & 0,56 \\
\hline 16 & PEREIRA & 0,88 & 32 & CAUCA & 0,54 \\
\hline
\end{tabular}

* Supereficiencia

En la Tabla 2 se puede observar que 12 universidades fueron eficientes, (valor de supereficiencia igual o superior a 1) en el año 2012: Militar, UNAD, Tolima, Distrital, UFPS-Ocaña, Magdalena, Guajira, Pedagógica, Cúcuta, Pacífico, Pamplona, Antioquia.

En el grupo de las Muy Pequeñas se observa que ambas representantes, Pacífico y UFPS-Ocaña, son eficientes, siendo Ocaña una de las cinco mejores del sistema. En el grupo de las universidades Pequeñas se aprecia que sólo dos resultan ser eficientes: UFPS-Cúcuta y Guajira. Las universidades ineficientes se encuentran entre 0.57 (Amazonas) y 0.98 (Popular). Militar y Magdalena son las únicas eficientes para el grupo de las universidades Medianas Bajas, siendo la Militar la más eficiente de todo el sistema con una supereficiencia de 2,93. Entre las universidades Medianas las eficientes fueron: UNAD, Tolima, Pedagógica y Pamplona. La UNAD es la segunda universidad más eficiente del sistema $(2,41)$. La única eficiente del grupo de universidades clasificadas como Grandes es la Distrital. Muy cerca de ser eficiente se encuentra la universidad del Valle, con una eficiencia de 0.97. La Universidad del Cauca solo alcanzó una eficiencia de 0.54 . Entre las universidades más grandes del sistema, solo la de Antioquia es eficiente.
Los indicadores:

valores objetivo y porcentaje de holgura

La metodología DEA permite obtener el valor objetivo que debe alcanzar cada UD en cada uno de los productos para alcanzar la eficiencia, información que junto a los valores observados permite calcular las deficiencias u holguras de las universidades. En la Tabla 3 se pueden apreciar las diferencias entre los valores observado y objetivo dadas en porcentajes de la siguiente forma: si el observado es superior al objetivo la diferencia porcentual es precedida del signo + e indica que la universidad sobrepasó el objetivo; si no está precedida del signo + el valor observado es inferior al objetivo y el valor es el porcentaje que requiere aumentar el indicador para alcanzarlo. Los espacios vacíos en la Tabla 3 indican que la universidad no presenta valores en el indicador correspondiente. 
Tabla 3. Porcentajes de mejora en el índice de formación 2012.

\begin{tabular}{|c|c|c|c|c|c|c|c|c|}
\hline \multirow{2}{*}{ IES } & \multicolumn{8}{|c|}{ PORCENT AJES ÍNDICE DE FORMACIÓN } \\
\hline & PPOS & PPRE & MPRE & MPOS & GPRE & GPOS & ECAES & PRIM \\
\hline PACÍFICO & . & +21 & 12 & 21 & 44 & . & 186 & 21 \\
\hline UFPS-OCAÑA & 115 & +84 & 14 & 91 & 45 & 10 & 71 & +57 \\
\hline SUCRE & 27 & 27 & 69 & 325 & 27 & . & 27 & 64 \\
\hline GUAJIRA & 33 & +61 & 6 & +21 & 34 & . & 226 & +48 \\
\hline NARIÑO & 13 & 13 & 72 & 81 & 62 & 59 & 13 & 13 \\
\hline COLMAYOR & 223 & 46 & 138 & 202 & 55 & 34 & 34 & 69 \\
\hline AMAZONAS & 130 & 75 & 76 & 97 & 75 & 75 & 154 & 147 \\
\hline LLANOS & 55 & 55 & 143 & 183 & 108 & 139 & 55 & 124 \\
\hline POPULAR & . & 2 & 2 &. & 2 & 4 & 23 & 87 \\
\hline UFPS-CÚCUTA & 17 & +38 & 38 & 21 & 9 & 3 & 38 & 25 \\
\hline CHOCÓ & 27 & 27 & 36 & 744 & 27 & 230 & 1283 & 27 \\
\hline SURCOLOMBIANA & 19 & 19 & 55 & 85 & 45 & 22 & 19 & 31 \\
\hline MAGDALENA & 54 & +58 & 40 & 24 & 77 & 77 & 8 & 77 \\
\hline CUNDINAMARCA & 253 & 76 & 124 & 594 & 131 & 76 & 76 & 76 \\
\hline CÓRDOBA & 77 & 77 & 77 & 154 & 201 & . & 128 & 77 \\
\hline MILITAR & +193 & 38 & 21 & +154 & 51 & 193 & 50 & 74 \\
\hline QUINDIO & 91 & 63 & 50 & 428 & 50 & 597 & 50 & 351 \\
\hline CARTAGENA & 13 & 13 & 36 & 137 & 108 & 181 & 13 & 13 \\
\hline PEDAGÓGICA & 24 & 14 & 0 & $\begin{array}{l}+47 \\
\end{array}$ & 47 & 3 & 47 & 8 \\
\hline PEREIRA & 13 & 13 & 63 & 44 & 70 & 178 & 13 & 54 \\
\hline CALDAS & 19 & 19 & 77 & 94 & 19 & 236 & 19 & 146 \\
\hline ATLÁNTICO & 24 & 24 & 36 & 288 & 49 & 139 & 24 & 24 \\
\hline TOLIMA & 59 & 15 & 89 & $\begin{array}{l}+\quad 89 \\
\end{array}$ & 89 & 89 & 89 & 39 \\
\hline PAMPLONA & 65 & 4 & 44 & 219 & 15 & 62 & 3 & 153 \\
\hline UNAD & 140 & 19 & $\begin{array}{r}+132 \\
\end{array}$ & +97 & 92 & 286 & 99 & 141 \\
\hline CAUCA & 85 & 85 & 131 & 264 & 85 & 304 & 104 & 304 \\
\hline UIS & 14 & 41 & 114 & 74 & 71 & 147 & 14 & 97 \\
\hline DISTRITAL & 8 & $\begin{array}{l}+6 \\
\end{array}$ & 90 & 27 & 41 & 88 & 88 & 279 \\
\hline UPTC & 135 & 35 & 77 & 56 & 47 & 35 & 36 & 127 \\
\hline VALLE & 3 & 3 & 127 & 20 & 74 & 124 & 3 & 149 \\
\hline ANTIOQUIA & 6 & 6 & 95 & 104 & 92 & 146 & 6 & 112 \\
\hline NACIONAL & 14 & 65 & 139 & 30 & 101 & 72 & 14 & 143 \\
\hline
\end{tabular}

En la Tabla 4 se presentan las universidades y su éxito o fracaso en cada uno de los indicadores analizados. Se destaca la Universidad del Magdalena que fue la única que en 2012 alcanzó los objetivos en todos los indicadores. Con siete objetivos alcanzados la Universidad del Tolima; con seis la Universidad Militar; con 5 las Universidades Distrital y Francisco de Paula Santander, seccional Cúcuta; con 4 objetivos las Universidades Pacífico, UFPSOcaña, Guajira, UNAD y Pedagógica; con tres objetivos alcanzados la Universidad de Antioquia y finalmente con dos objetivos alcanzados la Universidad de Pamplona.

Las universidades que no alcanzaron su objetivo en ningún indicador fueron: Sucre, Nariño, Colegio Mayor, Amazonas, Llanos, Popular, Chocó, Surcolombiana, Cundinamarca, Córdoba, Quindío, Cartagena, Pereira, Caldas, Atlántico, Cauca, UIS, UPTC, Valle y Nacional, más de la mitad del grupo evaluado. 
$\mathrm{Al}$ analizar cada indicador respecto al número de universidades que alcanzaron el objetivo de ese indicador, nos encontramos que los indicadores con menos éxito fueron los asociados con los posgrados, tanto el número de programas como el número de graduados, solo 5 universidades fueron exitosas. Seis universidades lograron cumplir los objetivos en graduados de pregrado. Siete universidades lograron el éxito en el número de estudiantes de primer semestre y matriculados en pregrado. Ocho universidades alcanzaron sus objetivos en matriculados en posgrado y en los programas de pregrado. El indicador con más éxito fue el de los estudiantes con resultados en el examen Saber-Pro superiores al percentil 75, nueve universidades lo alcanzaron.

Tabla 4. Las universidades y los indicadores en los cuales lograron o no lograron sus objetivos en 2012.

\begin{tabular}{|c|c|c|c|c|c|c|c|c|c|c|c|c|c|c|c|c|c|}
\hline IES & & $\begin{array}{l}\frac{u}{\alpha} \\
\frac{\alpha}{\alpha}\end{array}$ & $\begin{array}{l}\frac{u}{\alpha \underline{\alpha}} \\
\frac{\underline{\alpha}}{\Sigma}\end{array}$ & $\begin{array}{l}\infty \\
0 \\
\text { On } \\
\Sigma\end{array}$ & $\begin{array}{l}\frac{w}{\alpha \frac{1}{0}} \\
\frac{0}{0}\end{array}$ & $\begin{array}{l}0 \\
0 \\
0 \\
0\end{array}$ & 岕 & $\frac{\Sigma}{\frac{\Sigma}{\alpha}}$ & IES & $\begin{array}{l}\text { ஜ } \\
\text { O } \\
\frac{0}{\alpha}\end{array}$ & $\frac{\frac{u}{\alpha}}{\frac{\alpha}{\alpha}}$ & $\begin{array}{l}\frac{u}{\alpha \underline{\alpha}} \\
\frac{\alpha}{\Sigma}\end{array}$ & $\begin{array}{l}\text { D } \\
\text { O } \\
\text { L }\end{array}$ & $\begin{array}{l}\underset{\sim}{\alpha} \\
\frac{\sim}{0}\end{array}$ & $\begin{array}{l}\text { ஸ口 } \\
\text { O } \\
\text { 웅 }\end{array}$ & 岕 & $\frac{\Sigma}{\frac{\Sigma}{\alpha}}$ \\
\hline paciirco & $x$ & $\cdots$ & $\cdots$ & $\bullet$ & $x$ & $x$ & $x$ & $\cdots$ & QUINDÍO & $x$ & $\mathrm{x}$ & $x$ & $x$ & $x$ & $x$ & $x$ & $x$ \\
\hline UFPS-OCAÑA & $x$ & $\cdots$ & $\cdots$ & $x$ & $x$ & $\cdots$ & $x$ & $\cdots$ & CARTAGENA & $\mathrm{x}$ & $x$ & $x$ & $x$ & $x$ & $x$ & $x$ & $x$ \\
\hline SUCRE & $x$ & $x$ & $x$ & $x$ & $x$ & $x$ & $x$ & $x$ & PEDAGÓGICA & $x$ & $\cdots$ & $x$ & $\cdots$ & $\cdots$ & $x$ & $\cdots$ & $x$ \\
\hline GUAJIRA & $\cdots$ & $\cdots$ & $x$ & $\cdots$ & $x$ & $x$ & $x$ & $\cdots$ & PEREIRA & $x$ & $x$ & $x$ & $\mathrm{X}$ & $x$ & $x$ & $\mathrm{x}$ & $x$ \\
\hline NARIÑO & $x$ & $x$ & $x$ & $x$ & $x$ & $x$ & $x$ & $\mathrm{x}$ & CALDAS & $\mathrm{x}$ & $\mathrm{x}$ & $x$ & $x$ & $x$ & $x$ & $x$ & $x$ \\
\hline COLMAYOR & $x$ & $x$ & $x$ & $x$ & $x$ & $x$ & $x$ & $x$ & ATLÁNTICO & $x$ & $\mathrm{x}$ & $x$ & $x$ & $x$ & $x$ & $x$ & $x$ \\
\hline AMAZONAS & $x$ & $x$ & $x$ & $x$ & $x$ & $x$ & $x$ & $x$ & TOLIMA & $x$ & $\cdots$ & $\cdots$ & $\cdots$ & $\cdots$ & $\cdots$ & $\cdots$ & $\cdots$ \\
\hline LLANOS & $x$ & $x$ & $x$ & $x$ & $x$ & $x$ & $x$ & $x$ & PAMPLONA & $\mathrm{x}$ & $x$ & $x$ & $x$ & $\cdots$ & $x$ & $\cdots$ & $x$ \\
\hline POPULAR & $x$ & $x$ & $x$ & $x$ & $x$ & $x$ & $x$ & $x$ & UNAD & $x$ & $x$ & $\cdots$ & $\cdots$ & $x$ & $x$ & $\cdots$ & - \\
\hline UFPS-CÚCUTA & $x$ & $\cdots$ & $\cdots$ & $\cdots$ & $\bullet$ & $x$ & $\cdots$ & $x$ & CAUCA & $\mathrm{x}$ & $x$ & $x$ & $x$ & $x$ & $x$ & $x$ & $x$ \\
\hline CHOCÓ & $x$ & $x$ & $x$ & $x$ & $x$ & $x$ & $x$ & $x$ & UIS & $x$ & $x$ & $x$ & $x$ & $x$ & $x$ & $x$ & $x$ \\
\hline SURCOLOMBIANA & $x$ & $x$ & $x$ & $x$ & $x$ & $\mathrm{X}$ & $x$ & $x$ & DISTRITAL & $\cdots$ & $\bullet$ & $x$ & $x$ & $\cdots$ & $\cdots$ & $\cdots$ & $x$ \\
\hline MAGDALENA & $\cdots$ & $\cdots$ & $\cdots$ & $\cdots$ & $\cdots$ & $\cdots$ & - & $\cdots$ & UPTC & $x$ & $x$ & $x$ & $x$ & $x$ & $x$ & $x$ & $x$ \\
\hline CUNDINAMARCA & $x$ & $x$ & $x$ & $x$ & $x$ & $x$ & $x$ & $x$ & VALLE & $x$ & $x$ & $x$ & $x$ & $x$ & $x$ & $x$ & $x$ \\
\hline CÓRDOBA & $x$ & $x$ & $x$ & $x$ & $x$ & $x$ & $x$ & $\mathrm{x}$ & ANTIOQUIA & $\cdots$ & $\bullet$ & $x$ & $x$ & $x$ & $x$ & $\cdots$ & $x$ \\
\hline MILITAR & $\bullet$ & $x$ & $\cdots$ & $\bullet$ & $x$ & $\bullet$ & $\bullet$ & $\cdots$ & NACIONAL & $\mathrm{x}$ & $x$ & $x$ & $x$ & $x$ & $x$ & $x$ & $\mathrm{x}$ \\
\hline
\end{tabular}

\section{Conclusiones}

Antes que nada, es pertinente resaltar que los resultados aquí obtenidos deben asumirse con cierta reserva, no solamente por las especificidades del modelo, tal como se explicó previamente, sino también por el hecho de que el análisis realizado se centra exclusivamente en los productos asociados a la misión de Formación utilizando para ello toda la capacidad disponible de las universidades, cuando parte de esta capacidad también sustenta las demás actividades de la universidad, tales como la Investigación, la Extensión Solidaria y los programas de Bienestar Universitario.

En términos generales, se puede decir que si bien es cierto que el Sistema Universitario Estatal ha venido mejorando sus indicadores de formación en los últimos años, todavía hay mucho por hacer. Algunas universidades, en particular, han mostrado altos niveles de eficiencia en buena parte del periodo considerado, en tanto que existen otras cuyos resultados todavía están lejanos de ser eficientes.
En lo que respecta específicamente a los indicadores de formación, los valores observados en el año 2012 para todo el SUE en su conjunto son inferiores a los valores objetivo obtenidos por el modelo. Solo una universidad alcanzó el valor objetivo en todos los indicadores, 4 en más de la mitad, 6 en la mitad o menos de los indicadores, las 21 universidades restantes no lograron el objetivo en ninguno de los indicadores. Los programas y graduados de posgrado fueron los que menos éxito obtuvieron, de hecho son muy pocos todavía los programas de maestría y doctorado $y$, por ende, el número de los graduados en las universidades públicas colombianas. De otro lado, el indicador de estudiantes con resultados en el examen Saber-Pro superiores al percentil 75 fue el que mostró mejores resultados, 7 universidades alcanzaron el valor objetivo.

Es interesante anotar que las universidades de tamaños medio o pequeño, son las que exhiben mejor rendimiento en los indicadores de formación, siendo las de mayor capacidad las de menor rendimiento. Estas diferencias pueden deberse al hecho que estas últimas son las universidades de mejor rendimiento en los procesos investigativos que consumen gran parte de los recursos universitarios. 


\section{Referencias bibliográficas}

Abbott, M., Doucouliagos, C. (2003). The efficiency of Australian Universities: A Data Envelopment Analysis. Economics of Education Review 22, 89-97.

Banker, R.D., Chang, H. (2004). The super-efficiency procedure for outlier identification, not for ranking efficient units. European Journal of Operational Research.

Beasley, J. (1995). Determining Teaching and Research Efficiencies. Journal of the Operational Research Society 46, 441-452.

Bougnol, M.L., Dulá, J. (2006). Validating DEA as a Ranking Tool: An Application of DEA to Assess Performance in Higher Education. Annals of Operations Research 145, 339-365.

Coll, V., y Blasco, O. (2006). Evaluación de la eficiencia mediante el Análisis Envolvente de Datos, introducción a los modelos básicos. Universidad de Valencia, Valencia, España.

Cook, W.D., Seiford, L.M. (2009). Data Envelopment Analysis (DEA)Thirty Years On. European Journal of Operations Research, 192, 117.

Cooper, W., Seiford, L. y Tone, K. (2007). Data Envelopment Analysis. Editorial Springer Science y Business Media, con sede en New York, USA. Second Edition.

Fuentes Pascual, R. (2000). Eficiencia de la gestión de los institutos públicos de bachiller de la provincia de Alicante. Tesis doctoral no publicada. Universidad de Alicante, España.

Gómez, J. (2001). La evaluación de la eficiencia en las universidades públicas es pañolas: ht t p://www.paginaaede.org/Murcia/E01.pdf. [consulta: agosto 27, 2010].

González, A. (2010). Evaluación de la eficiencia de las Universidades Estatales Colombianas. Tesis de maestría en estadística no publicada. Universidad de los Andes, Mérida, Venezuela.

Johnes, J. (2006). Data Envelopment Analysis and Its Application to the Measurement of Efficiency in Higher Education. Economics of Education Review 25, 273-288.

Johnes, J. (2006). Measuring Teaching Efficiency in Higher Education An Application of Data Envelopment Analysis to Economics Graduates from University 1993. European Journal of Operations Research 174, 443-456.

Jones, J., Yu, L. (2008). Measuring the Research Performance of Chinese Higher Education Institutions Using Data Envelopment Analysis. China Economic Review 19,679-696.
Kuah, C.T., Wong, K.Y. (2011). Efficicency assessment of universities through data envelopment analysis. Procedia Computer Science.

Liu, Wen-Bin, Wongchai Anupong, Peng, Ke-Chung (2011). Adopting Super-efficiency and Tobit Model on Analyzing the Efficiency of Teacher's Colleges in Thailand.

Mercado, E., Díaz, E. y Diana, F. (1998). Productividad. Base de la competitividad. Caps 4 y 8 . Editorial Limusa, México.

Ministerio de Educación Nacional (1992). Ley 30 por la cual se organiza el servicio público de la Educación Superior, Bogotá.

Pedraja, F., Salinas, J., Smith,P. (1994). La Restricción de las Ponderaciones en el Análisis Envolvente de Datos: Una Fórmula para Mejorar la Evaluación de la Eficiencia. Investigaciones Económicas, Vol. XVIII (2), Mayo, pp. 365-380.

Pérez, M. (2010). Evaluación de Indicadores de Gestión en las universidades públicas Colombianas: Una aplicación de modelos de Ecuaciones Estructurales. Tesis de maestría en estadística no publicada. Universidad de los Andes, Mérida, Venezuela.

Pino, J.L., Solís, F., Delgado, M., Barea, R. (2010). Evaluación de la eficiencia de grupos de investigación mediante análisis envolvente de datos (DEA). El profesional de la información, v. 19 n.2, pp. 160167.

Quindós, M., Rubiera, F., Vicente, M. (2002). Análisis Envolvente de Datos: Una aplicación al sector de los servicios avanzados a las empresas del principado de Asturias.

Soto, J., Arenas, Wilson. y Trejos, C. (2005). La Producción Académica como uno de los indicadores del desempeño de las Universidades Públicas Colombianas desde la perspectiva del Análisis Envolvente de Datos. Scientia Et. Technica, Vol. XI, Núm. 28, pp. 109-114.

Torres, Y., Yáñez, G. (2012) Construcción de un modelo dinámico de indicadores para el Sistema Universitario Estatal. Entornos, Núm. 24, Edición Especial, Neiva.

Yáñez, G. y Torres, J. (2009). Análisis de los Indicadores de Gestión en la Universidad Industrial de Santander en el período 2003-2008. Publicado por la Asociación de Profesores de la Universidad Industrial de Santander, Bucaramanga, Colombia.

Yáñez, G. (2010). Análisis comparativo de los Indicadores de Gestión de las instituciones de educación superior de mayor tamaño en el período 2003-2008. Publicado por la Asociación de Profesores de la Universidad Industrial de Santander, Bucaramanga, Colombia. 